\title{
The Same Allele of Translation Initiation Factor 4E Mediates Resistance Against Two Potyvirus spp. in Pisum sativum
}

\author{
M. Bruun-Rasmussen, I. S. Møller, G. Tulinius, J. K. R. Hansen, O. S. Lund, and I. E. Johansen \\ Department of Genetics and Biotechnology, Faculty of Agricultural Sciences, University of Aarhus, Thorvaldsensvej 40, \\ DK-1871 Frederiksberg C, Denmark
}

Submitted 22 March 2007. Accepted 14 May 2007.

\begin{abstract}
Pathogenicity of two sequenced isolates of Bean yellow mosaic virus (BYMV) was established on genotypes of Pisum sativum $\mathrm{L}$. reported to carry resistance genes to BYMV and other potyviruses. Resistance to the white lupin strain of BYMV (BYMV-W) is inherited as a recessive gene named $w l v$ that maps to linkage group VI together with other Potyvirus resistances. One of these, sbm1, confers resistance to strains of Pea seedborne mosaic virus and previously has been identified as a mutant allele of the eukaryotic translation initiation factor $4 \mathrm{E}$ gene $(e I F 4 E)$. Sequence comparison of $e I F 4 E$ from BYMV-W-susceptible and -resistant $P$. sativum genotypes revealed a polymorphism correlating with the resistance profile. Expression of eIF4E from susceptible plants in resistant plants facilitated BYMV-W infection in inoculated leaves. When cDNA of BYMV-W was agroinoculated, resistance mediated by the wlv gene frequently was overcome, and virus from these plants had a codon change causing an Arg to His change at position 116 of the predicted viral genome-linked protein (VPg). Accordingly, plants carrying the $w l v$ resistance gene were infected upon inoculation with BYMV-W derived from cDNA with a His codon at position 116 of the VPg coding region. These results suggested that VPg determined pathogenicity on plants carrying the $w l v$ resistance gene and that wlv corresponded to the $s b m 1$ allele of $e I F 4 E$.
\end{abstract}

Additional keywords: Pea early browning virus.

Eukaryotic translation initiation factor $4 \mathrm{E}$ (eIF4E) and its isoform eIF(iso)4E play a key role during virus infection in plants (Robaglia and Caranta 2006). Most examples are known from the family Potyviridae; however, in the family Bromoviridae, Cucumber mosaic virus infection also has been demonstrated to rely on eIF4E as well as eIF4G (Yoshii et al. 2004). Accordingly, variants of $e I F 4 E$ with a few codon differences

Corresponding author: I. E. Johansen; E-mail: e.johansen@dias.kvl.dk

Current address for I. S. Møller: Australian Centre for Plant Functional Genomics, Private Mail Bag 1, Glen Osmond, SA 5064, Australia.

Current address for O. S. Lund: Department of Plant Biology, Faculty of Life Sciences, University of Copenhagen, Thorvaldsensvej 40, DK-1871 Frederiksberg C, Denmark.

Nucleotide sequence data for the white lupin strain of Bean yellow mosaic virus and Pisum sativum eIF4E cDNAs are available in the GenBank database under accession numbers DQ641248, DQ641470, DQ641471, DQ641472, DQ641473, and DQ641474. have been shown to confer resistance to infection, which is inherited as a recessive character (Gao et al. 2004b; Nicaise et al. 2003; Ruffel et al. 2002; Stein et al. 2005). Virus strains that can overcome recessive resistance genes have been identified in several Potyvirus spp. In most cases, breaking of resistance has been associated with mutations in the region encoding the genome-linked virus protein (VPg) (Ayme et al. 2006; Borgstrøm and Johansen 2001; Moury et al. 2004; Nicolas et al. 1997; Schaad et al. 1997). In at least three examples where breaking of resistance is associated with $\mathrm{VPg}$, the resistance gene was confirmed to be associated with mutations in $e I F 4 E$, namely the genes sbml in Pisum sativum (Borgstrøm and Johansen 2001; Gao et al. 2004b), pvr2 in pepper (Moury et al. 2004; Ruffel et al. 2002), and rym4 in barley (Kuhne et al. 2003; Stein et al. 2005). Therefore, it is likely that Potyvirus infection depends on interaction between host eIF4E and viral $\mathrm{VPg}$, as was demonstrated recently between VPg precursors and eIF(iso) $4 \mathrm{E}$ in planta (Beauchemin et al. 2007). Viruses with mutations in VPg often overcome resistance to virus accumulation (Nicaise et al. 2003), cell-to-cell movement (Gao et al. 2004b; Nicolas et al. 1997), or long-distance movement (Rajamäki and Valkonen 1999; Schaad et al. 1997), suggesting multiple functions of VPg. Though it is not known whether eIF4E plays a role in all these resistances, the interaction between VPg and eIF4E has been suggested to play a role in the early steps of infection during translation of virus proteins, viral replication, intracellular trafficking, and cell-to-cell movement (Robaglia and Caranta 2006).

In $P$. sativum, recessive resistance genes to several potyviruses have been mapped genetically to linkage groups II and VI (Provvidenti and Hampton 1991). The genes that map to linkage group II control resistance to Bean common mosaic virus (bcm), Clover yellow vein virus (ClYVV, cyvl), Bean yellow mosaic virus (BYMV, mo), Watermelon mosaic virus 2 ( $m o$ ), pea mosaic virus ( $p m v)$, and Pea seedborne mosaic virus (PSbMV, sbm2). The genes that map to linkage group VI show resistance to ClYVV (cyv2), PSbMV (sbml), and white lupin mosaic virus (wlv) (Provvidenti and Hampton 1991). Pea mosaic virus and white lupin mosaic virus are now considered as strains of BYMV (Shukla et al. 1994). In this article, we will refer to white lupin mosaic virus as BYMV-W. The pathotypespecific resistance genes $s b m 1$ and $s b m 2$ that affect PSbMV have been studied in some detail. Gene $s b m 1$ is an allele of eIF4E (Gao et al. 2004b), whereas sbm2 maps close to the isoform of eIF4E (eIF(iso)4E) (Gao et al. 2004a). The viral pathogenicity determinants toward $s b m 1$ and $s b m 2$ have been mapped to the regions encoding VPg and P3, respectively (Hjulsager et al. 2002). The identities of the other Potyvirus resistance genes 
in $P$. sativum remain to be identified. It is still not known whether the closely linked resistances cyvl, mo, pmv, and $s b m 2$ in linkage group II, as well as $c y v 2$, sbml, and $w l v$ in linkage group VI, are separate genes or the same gene with alleles of different specificity.

This study focuses on resistance to BYMV, which was first described by Yen and Fry (1956). The resistance gene mo was genetically characterized in $P$. sativum cv. Bonneville using an unspecified isolate of BYMV (Schroeder and Provvidenti 1971) and mapped to linkage group II (Marx and Provvidenti 1979). A second recessive resistance gene, $w l v$, conferring specific resistance to BYMV-W, was described in $P$. sativum plant introduction (PI) line PI193835 and mapped to linkage group VI (Provvidenti and Hampton 1993). BYMV-W was found to overcome resistance in Bonneville (Provvidenti and Hampton 1993) whereas resistance in PI193835 was overcome by an unspecified isolate of BYMV (Provvidenti and Hampton 1991).

Here, we describe the response of $P$. sativum genotypes to BYMV strains BYMV-S (Guyatt et al. 1996) and BYMV-W (Hampton et al. 1992) and analyze the role of eIF4E in resistance. Results imply that $w l v$ gene-mediated resistance to BYMV-W and sbml gene-mediated resistance to PSbMV is controlled by the same allele of $e I F 4 E$ and that a mutation in VPg allows BYMV-W to overcome resistance.

\section{RESULTS}

\section{BYMV isolates $S$ and $W$ display differential}

infection profiles on a panel of $P$. sativum genotypes.

The BYMV isolates BYMV-S (Randles et al. 1980) and BYMV-W (Hampton et al. 1992) were selected to initiate a study of BYMV resistance in P. sativum. BYMV-S originates from Vicia faba and was reported to be sensitive to an unspecified resistance in P. sativum (Randles et al. 1980), whereas BYMV-W originates from Lupinus albus and is sensitive to resistance mediated by the gene wlv (Provvidenti and Hampton 1993). The sequence of BYMV-W was determined in this study (DQ641248) and Guyatt and associates (1996) published the sequence of BYMV-S. Initially, BYMV-S and BYMV-W were screened on a panel of $P$. sativum genotypes with resis-

Table 1. Response of Pisum sativum genotypes to Bean yellow mosaic virus (BYMV) isolates $\mathrm{W}$ and $\mathrm{S}$ and Pea seedborne mosaic virus (PSbMV) isolates DPD1, L1, NEP1, and NY

\begin{tabular}{|c|c|c|c|c|}
\hline \multirow[b]{2}{*}{ Virus isolate } & \multicolumn{4}{|c|}{$P$. sativum genotype ${ }^{a}$} \\
\hline & $\begin{array}{c}\text { Bonneville } \\
\text { DSP } \\
\text { PI347422 } \\
\text { PI347484 }^{\text {b }}\end{array}$ & $\begin{array}{l}\text { JI2009 } \\
\text { Brutus }\end{array}$ & PI269818 & $\begin{array}{c}\text { PI193835 } \\
\text { PI347464 } \\
\text { JI1405 } \\
\text { PI347492 }^{\text {c }}\end{array}$ \\
\hline BYMV-W & $\mathrm{S}$ & $\mathrm{S}$ & $\mathrm{S}$ & $\mathrm{R}$ \\
\hline BYMV-S & $\mathrm{R}$ & $\mathrm{S}$ & $\mathrm{S}$ & $\mathrm{S}$ \\
\hline PSbMV-DPD $1^{\mathrm{d}}$ & $\mathrm{S}$ & $\mathrm{S}$ & $\mathrm{R}$ & $\mathrm{R}$ \\
\hline PSbMV-L1 & $\mathrm{R}$ & $\mathrm{S}$ & $\mathrm{R}$ & $\mathrm{R}$ \\
\hline PSbMV-NEP1 & $\mathrm{R}$ & $\mathrm{S}$ & $\mathrm{S}$ & $\mathrm{R}$ \\
\hline PSbMV-NY & $\mathrm{S}$ & $\mathrm{S}$ & $\mathrm{S}$ & $\mathrm{R}$ \\
\hline
\end{tabular}

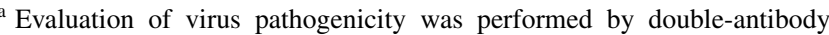
sandwich enzyme-linked immunosorbent assay (DAS-ELISA) of virus coat protein in uninoculated upper leaves 3 weeks postinoculation. DSP = Dark-skinned perfection and $\mathrm{PI}=$ plant introduction. $\mathrm{S}=$ susceptible: DAS-ELISA absorbance at $405 \mathrm{~nm}\left(\mathrm{~A}_{405}\right)$ of 80 to $100 \%$ of the tested plants was at least five times higher than the mean $\mathrm{A}_{405}$ of uninoculated controls. $\mathrm{R}=$ resistant: $\mathrm{A}_{405}$ of 80 to $100 \%$ of the tested plants were less than five times higher than the mean $\mathrm{A}_{405}$ of uninoculated controls.

${ }^{\mathrm{b}}$ Resistance gene mo originally was characterized in $P$. sativum cv. Bonneville (Schroeder and Provvidenti 1971).

${ }^{c}$ Resistance gene $w l v$ originally was characterized in P. sativum PI193835 (Provvidenti and Hampton 1993).

${ }^{d}$ Data on PSbMV (Hjulsager et al. 2002; Olsen and Johansen 2001). tance to potyviruses, and all have been tested previously for resistance to PSbMV (Gao et al. 2004b; Olsen and Johansen 2001). Virus isolates BYMV-S and BYMV-W were maintained in $P$. sativum cv. Brutus and sap of infected leaves was used to inoculate 2 -week-old $P$. sativum plants. Virus accumulation in uninoculated upper leaves was determined by double-antibody sandwich enzyme-linked immunosorbent assay (DAS-ELISA) 3 weeks postinoculation (wpi) using antibodies that were raised against purified BYMV-S or BYMV-W particles. Plants were considered infected if DAS-ELISA absorbance at $405 \mathrm{~nm}\left(\mathrm{~A}_{405}\right)$ was at least five times higher than the mean $\mathrm{A}_{405}$ of uninoculated controls. Judged according to this criterion, four P. sativum lines displayed resistance to BYMV-S but were susceptible to BYMV-W. These lines include cv. Bonneville, cv. Dark-skinned perfection (DSP), PI347422, and PI347484. The opposite response was observed in lines PI193835, PI347464, PI347492, and JI1405 that all displayed resistance to BYMV-W but were susceptible to BYMV-S. The line PI269818, which has been reported to carry resistance to an unspecified isolate of BYMV (Provvidenti 1987), was susceptible to both BYMV-S and BYMV-W. Based on this screen, we selected the following genotypes for further analysis of BYMV resistance: Brutus and JI2009 (cv. Scout), which were susceptible to both BYMV-S and BYMV-W and to PSbMV (Olsen and Johansen 2001); cvs. Bonneville and DSP, which both displayed resistance to BYMV-S and were susceptible to BYMV-W; and PI193835 and PI347464, which both were susceptible to BYMV-S and displayed resistance to BYMV-W and to PSbMV (Olsen and Johansen 2001). Cv. Bonneville originally was used to determine inheritance of resistance gene mo (Schroeder and Provvidenti 1971). PI193835 was included, although some individuals of PI193835 displayed DAS-ELISA readings indicating that the plants were infected by BYMV-W, because PI193835 originally was used to determine inheritance of resistance gene $w l v$ (Provvidenti and Hampton 1993). Pathogenicity profiles of BYMV and PSbMV isolates are summarized in Table 1.

\section{Sequence differences in $e I F 4 E$ correlate} with the infection profile of BYMV-W.

Gene $s b m 1$, mediating resistance against PSbMV, and gene $w l v$, mediating resistance against BYMV-W, both map to linkage group VI (Provvidenti and Hampton 1991). It has been shown previously that resistance to PSbMV in PI193835 is due to codon differences in $e I F 4 E$ (Gao et al. 2004b). To determine whether a similar correlation existed between the $e I F 4 E$ coding sequence and BYMV-W infection, eIF4E cDNAs from BYMV-W -susceptible Bonneville, DSP, PI269818, and Brutus and BYMV-W-resistant PI193835 and PI347464 were cloned and sequenced. An alignment of the predicted amino acid sequences with previously published sequences of JI2009 and JI1405 is shown in Figure 1. The alignment identified a correlation between Leu62, Asp73, Arg107, and Lys169 and resistance to BYMV-W. There was no correlation between BYMV-S resistance and specific amino acids in eIF4E of BYMV-S-resistant cvs. Bonneville and DSP. This observation agreed well with the assumption that resistance to BYMV-S is controlled by linkage group II gene mo in Bonneville and DSP (Provvidenti and Hampton 1991). However, sequencing $P$. sativum cDNA of eIF(iso) $4 E$ did not reveal differences correlating to BYMV-S resistance (DQ778076, DQ778077, DQ778078, and Bruun-Rasmussen; unpublished data).

\section{Expression of eIF4E from JI2009 leads to increased susceptibility to BYMV-W in plants carrying $w l v$.}

To determine whether resistance gene $w l v$ corresponds to eIF4E, we decided to test whether BYMV-W infection in re- 
sistant PI193835 could be complemented by coexpression of eIF4E from BYMV-susceptible $P$. sativum (S-eIF4E). To express the S-eIF4E in PI193835, we inserted the eIF4E coding region from $P$. sativum JI2009 into RNA2 of an expression vector based on Pea early browning virus (PEBV, genus Tobravirus) (Constantin et al. 2004). Two controls were included, one with an insertion of the sequence encoding eIF4E from PI193835 (R-eIF4E) and the other with the coding region of green fluorescent protein (GFP). The three recombinant PEBV RNA2 plasmids were named pCAPE2-S-eIF4E, pCAPE2-ReIF4E, and pCAPE2-GFP. To allow agroinoculation of BYMV$\mathrm{W}$ together with recombinant PEBV vectors, the BYMV-W sequence was cloned into a binary pCAMBIA vector to generate pCABYMV-W.

For the complementation analysis, 2-week-old plants of BYMV-W-resistant PI193835 and BYMV-W-susceptible Bonneville were agroinoculated with pCABYMV-W, pCAPE1 that carries cDNA of PEBV RNA1, and pCAPE2-R-eIF4E, pCAPE2-S-eIF4E, or pCAPE2-GFP. Vectors pCABYMV-W and pCAPE1 were present in all agroinoculations and, therefore, only the pCAPE2 derivative will be mentioned in the following descriptions of experiments. In an initial experiment, infection by BYMV-W was determined by DAS-ELISA 5 days postinoculation (dpi) on an inoculated leaf of each plant. Samples were rated positive if the $\mathrm{A}_{405}$ reading was at least five times higher than the mean $\mathrm{A}_{405}$ of uninoculated controls. On PI193835 coinoculated with pCAPE2-S-eIF4E, 9 of 17 leaves tested positive, whereas 0 of 11 leaves and 2 of 18 leaves tested positive on plants coinoculated with pCAPE2-R-eIF4E and pCAPE2-GFP, respectively. All Bonneville plants tested positive regardless of the coinoculated pCAPE2 vector. However, because the $\mathrm{A}_{405}$ readings generally were low, we decided to analyze inoculated leaves 8 dpi and also to analyze uninoculated leaves 3 wpi. Results of two further experiments (Fig. 2A) showed that $76 \%$ of leaves from PI193835 coinoculated with pCAPE2-S-eIF4E tested positive for BYMV-W, with an average $\mathrm{A}_{405}$ reading of 2.0 . In leaves coinoculated with pCAPE2-GFP or pCAPE2-R-eIF4E, less than $10 \%$ of the samples tested positive for BYMV-W, with an average $\mathrm{A}_{405}$ reading of 0.36 . A $\chi^{2}$ test confirmed that coinoculation with pCAPE2$\mathrm{S}$-eIF4E resulted in significantly higher fraction of infected plants than coinoculation with pCAPE2-R-eIF4E or pCAPE2GFP $(P<0.001)$. In Bonneville, DAS-ELISA tests showed that BYMV-W could be detected in inoculated leaves 8 dpi in all but one plant regardless of the coinfiltrated pCAPE2 vector. Thus, results of DAS-ELISA tests of inoculated leaves indicated that BYMV-W could be complemented in PI193835 by coinoculation with pCAPE2-S-eIF4E, but not by coinoculation with pCAPE2-GFP or pCAPE2-R-eIF4E.

Six PI193835 plants coinoculated with pCAPE2-GFP or pCAPE2-R-eIF4E displayed DAS-ELISA readings more than five times those of the uninoculated controls, indicating that the plants were becoming infected. This was supported by DAS-ELISA data from uninoculated upper leaves sampled 3 wpi. At this time, $71 \%$ of the plants coinoculated with pCAPE2-S-eIF4E, 60\% coinoculated with pCAPE2-GFP, and $40 \%$ coinoculated with pCAPE2-R-eIF4E were DAS-ELISA positive, all with comparable $\mathrm{A}_{405}$ readings. Though the percent-
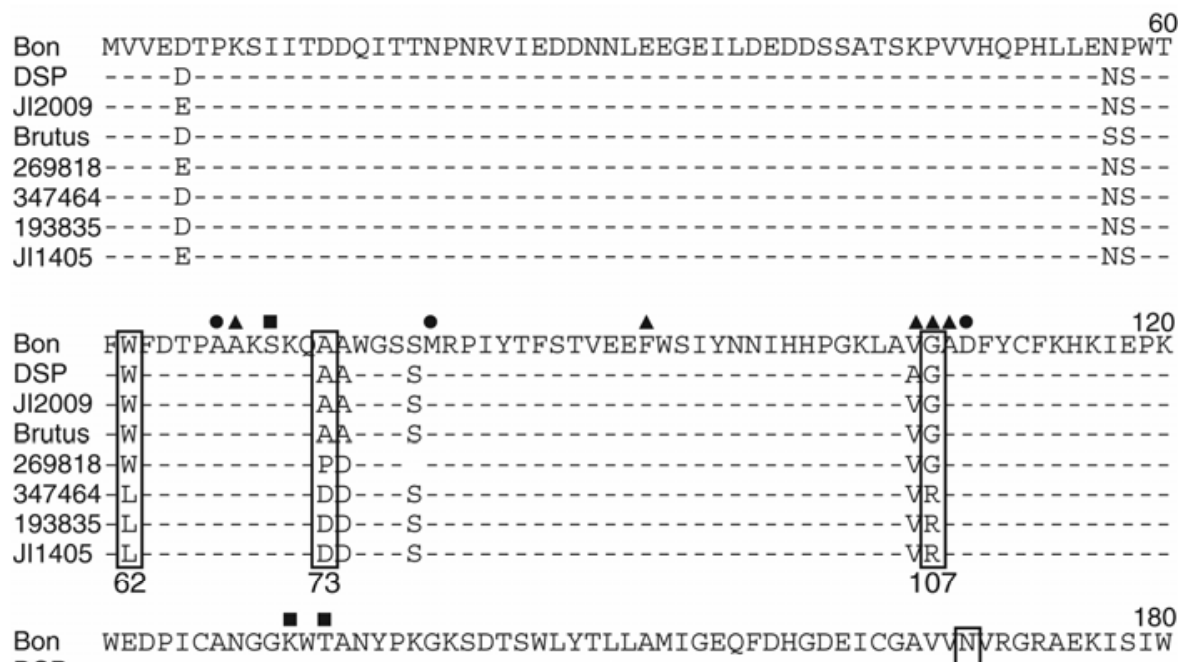
DSP

Brutus - - - - - 269818- - - 347464-- - - - - - - - - - - - - - L - - - - - - - - - -

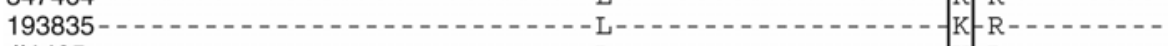

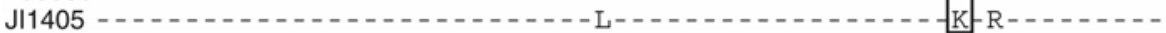
169

Bon TKNÂSNEAAQVSIGKQWKEFLDYNETMGFIFHDDARKLDRNAKNKYVV

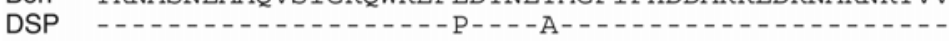

JI2009 - - - - - - - - - - - - - L- - - T- - - - - - - - - -

Brutus - - - - - - - - - - - - - L- - - T- - - - - - - - - - - - -

269818--- - - - - - - - - - - L- - - T- -

347464-- - - - - - - - - - L- - - T- - - - - - - - - -

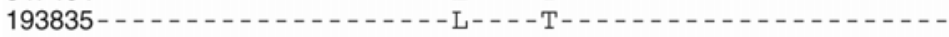

J1405 - - - - - - - - - - - - - L- - - T- - - - - - - - -

Fig. 1. Alignment of predicted protein sequences of eIF4E from Bean yellow mosaic virus (BYMV)-W-susceptible Bonneville (Bon), DSP, JI2009, Brutus, and PI269818 and BYMV-W-resistant PI347464, PI193835, and JI1405. Hyphens mark amino acids that are conserved in Pisum sativum eIF4E, and open boxes indicate differences correlating with resistance to BYMV-W. The locations associated with resistance to Lettuce mosaic virus. (Nicaise et al. 2003), Potato virus $Y$ (Ruffel et al. 2002), and BaMMV (Stein et al. 2005) are indicated with triangles, circles, and squares, respectively. 
age of DAS-ELISA-positive plants coinoculated with pCAPE2S-eIF4E was higher than plants coinoculated with pCAPE2GFP and pCAPE2-R-eIF4E, the difference was not significant $(0.1>P>0.05)$.

\section{The VPg sequence of virus isolated from PI193835.}

We speculated that DAS-ELISA-positive samples of upper, uninoculated PI193835 leaves observed 3 wpi could be due to the appearance of BYMV-W variants that break resistance. In the initial analysis of BYMV-W, a few PI193835 plants displayed DAS-ELISA readings above the threshold set to five times the uninoculated controls. However, inoculation by agroinfiltration may increase the opportunity for mutants to arise during transcription from the binary vector. Because there are several examples that mutations in the VPg coding region are
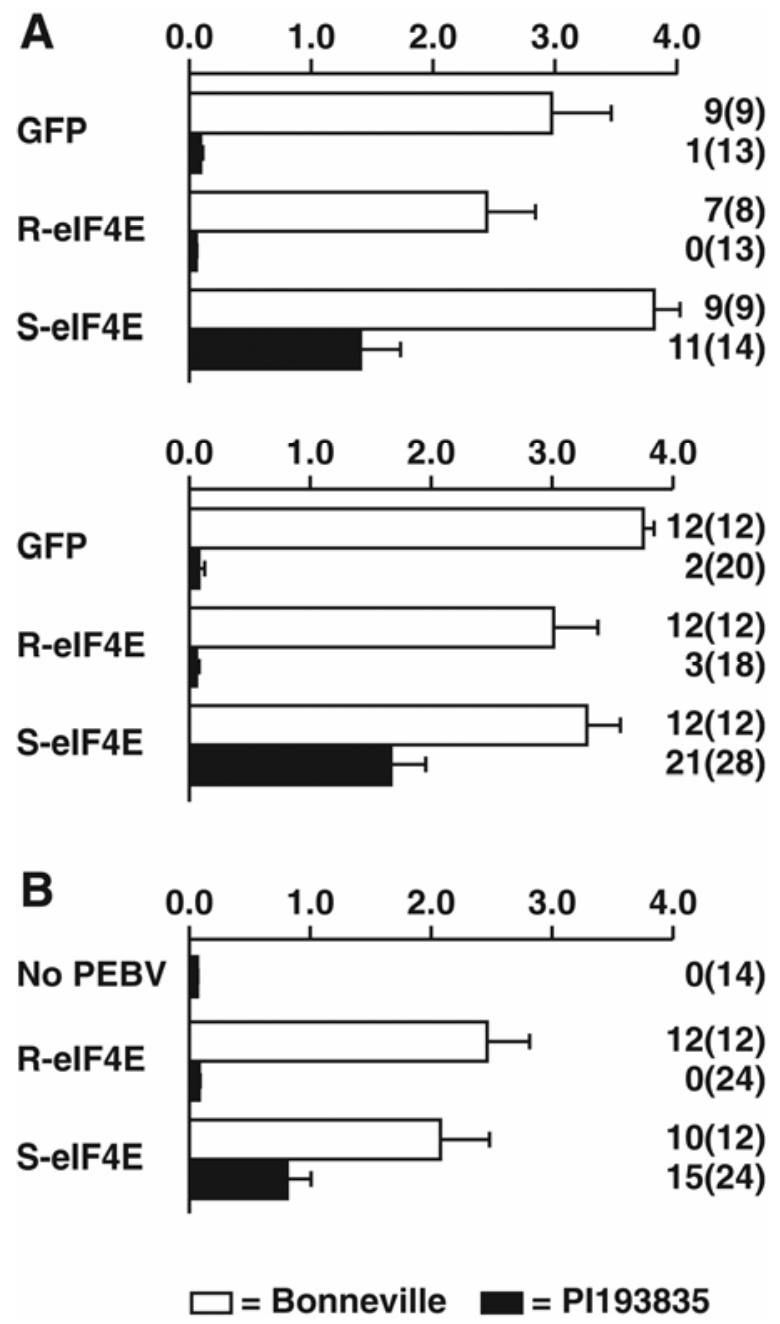

Fig. 2. Complementation of Bean yellow mosaic virus (BYMV)-W by eIF4E expressed from a pea early browning virus (PEBV) vector. BYMV-W infection was assayed by double-antibody sandwich enzyme-linked immunosorbent assay (DAS-ELISA) of coat protein in inoculated leaves 8 days postinoculation. Columns present mean DAS-ELISA readings and bars standard error of mean. Numbers next to the columns indicate number of samples rated as positive with DAS-ELISA absorbance at $405 \mathrm{~nm}\left(\mathrm{~A}_{405}\right) \geq 5 \times$ mean $\mathrm{A}_{405}$ of uninoculated controls (number of samples tested). A, Results of two independent experiments in which leaves of Pisum sativum Bonneville (susceptible to BYMV-W) and PI193835 (resistant to BYMV-W) were inoculated by agroinfiltration of pCABYMV-W together with pCAPE1 and pCAPE2-GFP (green fluorescent protein), pCAPE2-R-eIF4E (R-eIF4E), or pCAPE2-S-eIF4E (S-eIF4E). B, Results of an experiment in which leaves of $P$. sativum Bonneville and PI193835 were inoculated by agroinfiltration of pCABYMV-W alone (No PEBV), pCABYMV together with pCAPE1 and pCAPE2-R-eIF4E, or pCAPE2-S-eIF4E. associated with overcoming recessive resistance to potyviruses, we decided to analyze this region of the BYMV-W sequence. For each treatment (coinoculation with pCAPE2-GFP, pCAPE2-S-eIF4E, and pCAPE2-R-eIF4E), RNA was extracted from uninoculated leaves of two Bonneville and four PI193835 plants that tested positive in DAS-ELISA 3 wpi. The VPg coding region was amplified by reverse-transcriptase polymerase chain reaction (RT-PCR) and the resulting PCR products were purified and sequenced. The VPg sequences from Bonneville plants were identical to the VPg sequence in the cDNA clone, whereas a unique $\mathrm{G}$ to $\mathrm{A}$ change was found in all 12 samples from PI193835. The $G$ to A change was found in virus from PI193835 regardless of the coinoculated pCAPE2 construct. The resulting CGC to CAC codon change resulted in an Arg to His change at position 116 in the predicted amino acid sequence of VPg. This suggested that BYMV-W had overcome resistance mediated by gene $w l v$ by adaptation of VPg to PI193835.

\section{BYMV-W with VPg codon 116 CGC accumulates in leaves coinoculated with pCAPE2-S-eIF4E.}

The elevated $\mathrm{A}_{405}$ readings in leaves of PI193835 coinoculated with pCAPE2-S-eIF4E could be a result of local accumulation of the original BYMV-S with an unchanged CGC Arg codon at position 116 of $\mathrm{VPg}$ or accumulation of the CAC variant. In the VPg coding region of BYMV-W, the Arg codon 116 is part of an HaeII restriction site (PuGCGCPy, Arg codon in bold), which was eliminated when the Arg codon mutated to an His codon (AGCACT, His codon in bold). To determine which variant accumulated in PI193835 leaves coinoculated with pCAPE2-S-eIF4E, 2-week-old plants of BYMV-Wresistant PI193835 and BYMV-W-susceptible Bonneville were coinoculated with pCAPE2-S-eIF4E. Plants coinoculated with pCAPE2-R-eIF4E or with pCABYMV-W alone served as controls. At 8 dpi, inoculated leaves were homogenized and half of the sample was analyzed by DAS-ELISA (Fig 2B) and half was used to extract RNA. In DAS-ELISA, 15 of 24 samples tested positive for BYMV CP in plants coinoculated with pCAPE2-S-eIF4E, while none of the samples from plants coinoculated with pCAPE2-R-eIF4E or inoculated with pCABYMV-W alone were positive in DAS-ELISA. RNA samples were DNAse treated to remove pCABYMV inoculum and RT-PCR was conducted using a primer set amplifying a 620bp band of the VPg coding region. This region was modified in pCABYMV by insertion of a 189-bp intron, and any residual DNA from the inoculum would give rise to an 809-bp PCR fragment (Fig. 3A and B, lane 11 and 12). Digestion of the 620-bp PCR product of the BYMV-W VPg coding region by HaeII was expected to result in two fragments of 330 and 290 bp if the CGC Arg codon was still present (Fig. 3B, lane 11). RT-PCR resulted in amplification of the expected 620-bp fragment from RNA of PI193835 coinoculated with pCAPE2-SeIF4E, but not from plants coinoculated with pCAPE2-ReIF4E (Fig. 3B, lanes 3 to 6 and 9 and 10) or plants inoculated with pCABYMV alone (data not shown). RT-PCR resulted in amplification of the expected 620-bp fragment from Bonneville, regardless of the coinoculated pCAPE2 construct (Fig. 3B, lanes 1 and 2 and 7 and 8). When PCR fragments were digested with HaeII, the 620-bp band was cleaved into fragments corresponding in size to the expected 330 and 290 bp (Fig. 3C). Sequence analysis of the 620-bp PCR fragment amplified from PI193835 plants coinoculated with pCAPE2-S-eIF4E confirmed that the sequence was identical to the VPg coding sequence in the cDNA clone. This experiment demonstrated that BYMV-W with an unmodified VPg sequence was complemented specifically by S-eIF4E before it adapts to PI193835 by mutation of VPg codon 116 from CGC (Arg) to CAC (His). 
Mutation of VPg codon 116 in BYMV-W is sufficient to overcome $w l v$ resistance.

To determine whether the single codon change at position 116 of the VPg coding region was responsible for breaking resistance in PI193835, we introduced this mutation to VPg of
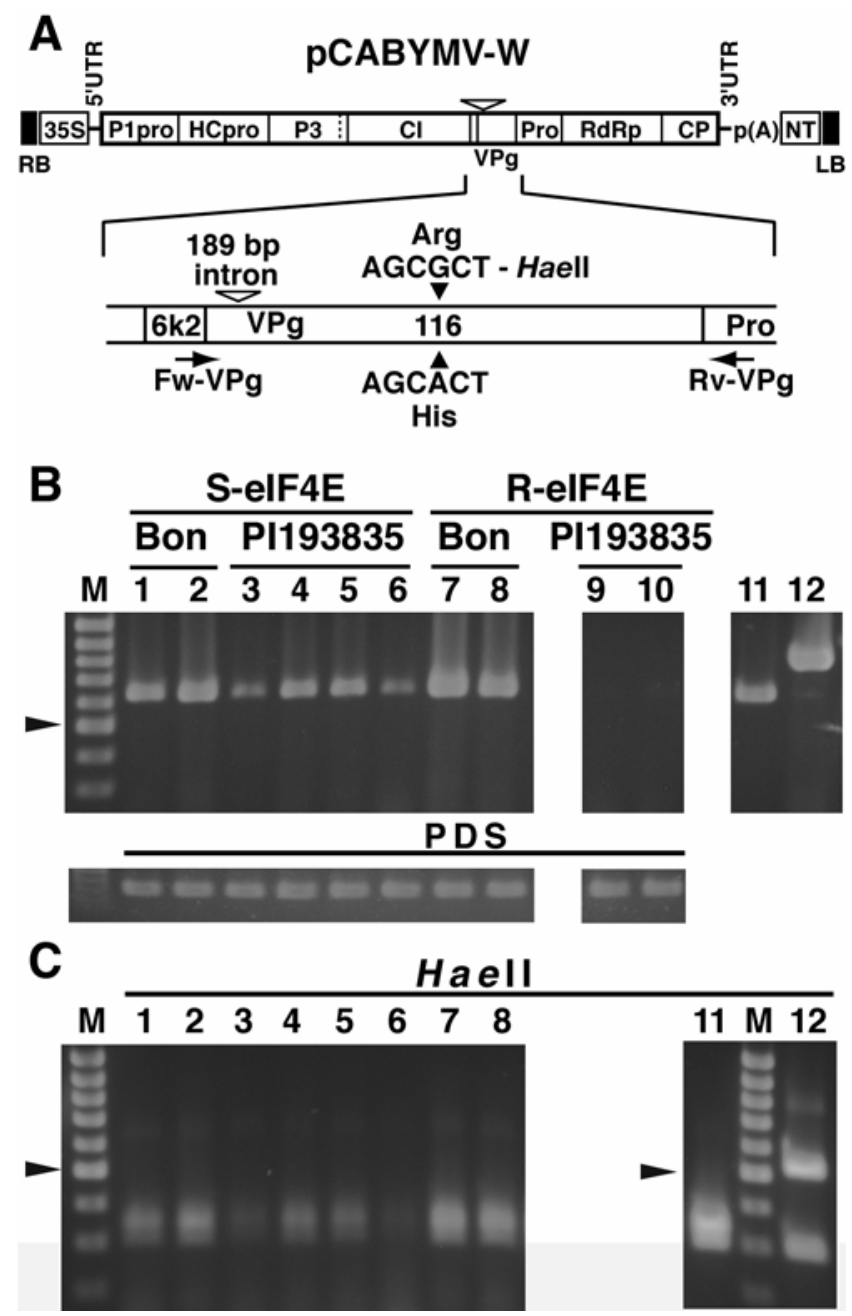

Fig. 3. Analysis of reverse-transcriptase polymerase chain reaction (RT$\mathrm{PCR})$ products of the viral genome-linked protein $(\mathrm{VPg})$ coding region from RNA extracted from leaves agroinfiltrated with pCABYMV together with pCAPE1 and pCAPE2-S-eIF4E or pCAPE2-R-eIF4E. A, T-DNA region of pCABYMV with an enlargement of the VPg coding region. Regions representing $5^{\prime}$ and $3^{\prime}$ untranslated regions are shown as horizontal lines, with the coding region as an open box with regions predicted to encode potyviral proteins P1pro, HCpro, P3, 6k1, CI, 6k2, Pro, RdRp, and CP (Revers et al. 1999). The intron (open triangle) that is absent in bean yellow mosaic virus (BYMV)-W RNA allowed PCR primers Fw-VPg and $\mathrm{Rv}-\mathrm{VPg}$ (arrows) to discriminate between fragments amplified from viral RNA and DNA inoculum. The polymorphy at codon 116 is displayed with the BYMV-W Arg codon in the context of an HaeII restriction site above and the mutated His codon below the enlarged VPg coding region. B, Agarose gel displaying RT-PCR products amplified by Fw-VPg and Rv-VPg on RT reactions of RNA extracted from leaves 8 days post inoculation with pCABYMV, pCAPE1, and pCAPE-S-eIF4E (lanes 1 to 6) or pCAPE2-ReIF4E (lanes 7 to 10). Lanes 1, 2, 7, and 8: RNA from BYMV-Wsusceptible P. sativum cv. Bonneville; lanes 3 to 6, 9, and 10: RNA from BYMV-W-resistant $P$. sativum PI193835. PCR products amplified from cDNA without (lane 11) and with (lane 12) an intron indicate the size of PCR products expected from RT-PCR of viral RNA and PCR of pCABYMV DNA inoculum, respectively. Lane M: 100-bp DNA ladder with 500-bp band marked by an arrowhead. PDS shows an RT-PCR amplification control corresponding to a 470-bp fragment of $P$. sativum phytoene desaturase. C, Agarose gel displaying HaeII digestion of RT-PCR products amplified by Fw-VPg and Rv-VPg. Lane numbering and markers are as in B.
BYMV-W, generating pBYMV-W $\mathrm{W}^{\mathrm{H} 116}$. To make a direct comparison of BYMV-W and BYMV-W ${ }^{\mathrm{H} 116}$ that was not influenced by the efficiency of inoculation of cDNA constructs, virus inocula were established by manual inoculation of susceptible cv. Brutus with plasmids pBYMV and pBYMV-W ${ }^{\mathrm{H} 116}$. Sap from these plants was used to inoculate BYMV-W-resistant PI193835 and PI347464 and BYMV-W-susceptible Brutus. Pathogenicity was assayed by DAS-ELISA 3 wpi on leaf samples from uninoculated leaves (Fig. 4A). Of the plants inoculated with BYMV-W ${ }^{\mathrm{H} 116}, 97 \%$ of PI193835 and PI347464 were positive in DAS-ELISA compared with $6 \%$ of plants inoculated with BYMV-W (Fig 4A), confirming that virus derived from a cDNA clone with this single codon change in VPg could overcome $w l v$ resistance. In a second experiment, virus derived from $\mathrm{pBYMV-}$ $\mathrm{W}^{\mathrm{H} 116}$ also was inoculated to Bonneville and DSP (Fig. 4B). All DSP and Bonneville plants tested positive in DAS-ELISA, suggesting that the change of VPg codon 116 to His (CAC) did not have a major effect on pathogenicity on Bonneville and DSP plants.

\section{DISCUSSION}

We have established the infection profile of two sequenced BYMV isolates, BYMV-S and BYMV-W, on a panel of $P$. sativum lines with reported resistance to several potyviruses, including BYMV and PSbMV (Olsen and Johansen 2001; Provvidenti and Hampton 1993). Two of these lines, Bonneville and PI193835, were used originally to map the resistance genes mo (Schroeder and Provvidenti 1971) and wlv (Provvidenti and

A A 405 sample / A 405 control

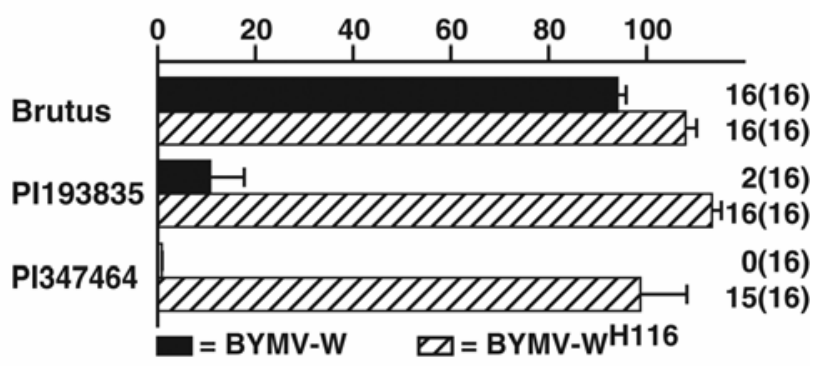

B

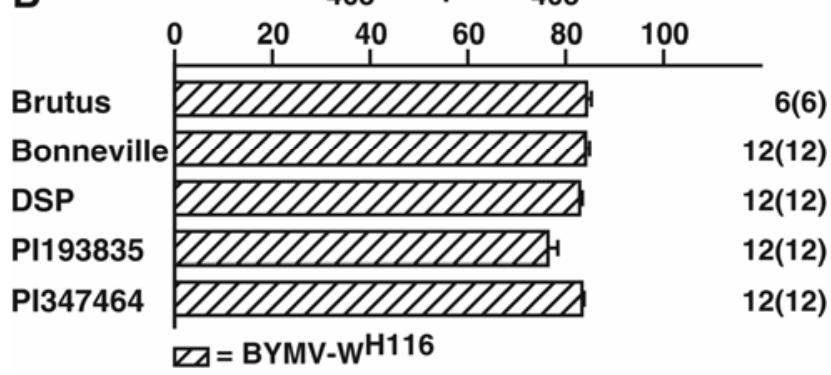

Fig. 4. Pathogenicity of viral genome-linked protein ( $\mathrm{VPg})$ mutant Bean yellow mosaic virus (BYMV)- $\mathrm{W}^{\mathrm{H} 116}$ in Pisum sativum. Columns represent mean double-antibody sandwich enzyme-linked immunosorbent assay (DAS-ELISA) absorbance at $405 \mathrm{~nm}\left(\mathrm{~A}_{405}\right)$ readings relative to the mean $\mathrm{A}_{405}$ reading of uninoculated controls and bars show standard error of mean. Numbers indicate samples rated as positive with DAS-ELISA $\mathrm{A}_{405}$ $\geq 5 \times$ mean $\mathrm{A}_{405}$ of uninoculated controls (number of samples tested). Samples were taken from uninoculated leaves 3 weeks post inoculation. A, Relative accumulation of BYMV coat protein in genotypes Brutus, PI193835, and PI347464 inoculated with BYMV-W (black bars) and BYMV-W ${ }^{\mathrm{H} 116}$ (hatched bars). B, Relative accumulation of BYMV coat protein in genotypes Brutus, Bonneville, DSP, PI193835, and PI347464 inoculated with BYMV- $\mathrm{W}^{\mathrm{H} 116}$. 
Hampton 1993) to linkage group II and VI, respectively. Genes mediating resistance to BYMV-W and PSbMV in linkage group VI originally were named $w l v$ and $s b m$, respectively, and $s b m$ was differentiated into $s b m 1, s b m 3$, and $s b m 4$ depending on the PSbMV pathotype (Provvidenti and Hampton 1991). Gao and associates (2004a) showed that $e I F 4 E$ colocalized with $s b m$ in linkage group VI and sequence analysis of $e I F 4 E$ that identified three alleles of eIF4E in P. sativum lines JI2009, PI193835, and PI269818. In complementation experiments, it was found that expression of eIF4E from susceptible JI2009 supported infection of PSbMV in PI193835 and PI269818 (Gao et al. 2004b). This led to a revision of the nomenclature. Thus, $e I F 4 E$ from PSbMV-susceptible JI2009 was designated Sbm1, eIF4E from PI193835 that is resistant to the four known pathotypes of PSbMV was designated sbml, and eIF4E from PI269818 that is overcome by PSbMV pathotypes P3 and P4 was designated $s b m 1^{1}$. The present study of resistance to BYMV-W in PI193835, which carries the gene traditionally known as $w l v$ in linkage group VI (Provvidenti and Hampton 1993), demonstrated that BYMV-W infection was complemented by expression of eIF4E corresponding to Sbml (Figs. 2 and 3). This suggested that $s b m l$ and $w l v$ correspond to the same allele of eIF4E. This parallels the situation in Capsicum spp., where resistances known as $p v r 1, p v r 2^{l}$, and $p v r 2^{2}$ were found to be alleles of $e I F 4 E$ conferring broad-spectrum or more specific resistance to viruses of the genus Potyvirus (Kang et al. 2005).

The $P$. sativum gene $s b m 1^{l}$ is an allele of eIF4E that confers specific resistance to some pathotypes of PSbMV (Gao et al. 2004b). PSbMV pathogenicity on $P$. sativum that carries $s b m l^{1}$ was associated with differences in the VPg coding region (Borgstrøm and Johansen 2001). In PSbMV, mutation of VPg codon 116, which is equivalent to codon 118 in BYMV-W, was sufficient of alter pathogenicity on plants carrying the $s b m 1^{1}$ allele of $e I F 4 E$; however, it was not investigated whether these mutations affected pathogenicity on plants carrying the $s b m 1 / w l v$ allele of eIF4E (Borgstrøm and Johansen 2001). Similarly, other resistances mediated by alleles of $e I F 4 E$ to other viruses in the family Potyviridae were overcome by virus isolates with mutations in the same part of the VPg coding region (Kuhne et al. 2003; Moury et al. 2004). In the present study, agroinoculation of BYMV-W from the binary plasmid pCABYMV resulted in the appearance of a resistance-breaking variant carrying a CGC (Arg) to CAC (His) codon change in the VPg coding region. This change was observed regardless of the coinoculated pCAPE2 construct, suggesting that, although S-eIF4E can complement BYMV-W in inoculated leaves (Fig. 3), this complementation was not sufficient to assist systemic infection by BYMV-W in competition with the resistance-breaking variant. It appeared that BYMV-W required only one nucleotide change to overcome resistance, because PI193835 and PI347464 plants inoculated with virus derived from $\mathrm{pBYMV}-\mathrm{W}^{\mathrm{H} 116}$ displayed $\mathrm{A}_{405}$ readings suggesting virus accumulation in uninoculated leaves (Fig. 4A). Of course, we cannot rule out the possibility that second-site compensatory mutations arose in the viruses during amplification in susceptible cv. Brutus. To minimize selective differentiation of BYMV-W and $\mathrm{pBYMV}-\mathrm{W}^{\mathrm{H} 116}$, both viruses were raised from cDNA clones and amplified in the same genetic background; however, it is possible that putative second-site mutations could be biased by the difference at VPg codon 116 in such a way that only virus derived from pBYMV-W ${ }^{\mathrm{H} 116}$ overcame resistance.

If breaking of resistance required only one nucleotide transition to change the Arg (CGC) codon to His (CAC), this may explain why the resistance-breaking isolate appeared frequently in plants infected by agroinoculation. Resistance breaking also was observed in plants inoculated by manual inoculation, but at a much lower frequency. This raises the possibility that agroinoculation may be an efficient way to provoke amino acid changes to challenge resistance, thereby enabling predictions on resistance durability, as suggested by Ayme and associates (2006).

Although virus derived from BYMV-W ${ }^{\mathrm{H} 116}$ overcame resistance in PI193835 and PI347464, pathogenicity on Bonneville and DSP was not compromised (Fig. 4B). In contrast, BYMV$\mathrm{S}$, which has a His codon (CAC) at position 116 of $\mathrm{VPg}$ and was infectious on PI193835 and PI347464, did not infect Bonneville and DSP. This would suggest that the mechanism of resistance mediated by $s b m 1 / w l v$ in PI193835 and PI347464 differed from $m o$ resistance in Bonneville and DSP. Supporting this interpretation, the pathogenicity determinant in PSbMV toward $s b m 2$, which is closely linked to mo (Provvidenti and Alconero 1988), was mapped to the $\mathrm{P} 3$ coding region (Johansen et al. 2001). The linkage between $\operatorname{eIF}$ (iso) $4 \mathrm{E}$, sbm2, and mo made $e I F$ (iso) $4 E$ a likely candidate for the potyvirus resistance gene in linkage group II of $P$. sativum (Gao et al. 2004a). Therefore, it was surprising that we did not identify any coding differences in the cDNA of $P$. sativum eIF(iso) $4 E$ correlating to the presence of mo resistance (M. Bruun-Rasmussen, unpublished data). Other candidates may be found among the translation initiation factors (Albar et al. 2006; Ruffel et al. 2006). In conclusion, our experiments provide a link between classical genetic studies and virological experiments (Hampton et al. 1992; Provvidenti and Hampton 1991, 1993) to specific alleles of $e I F 4 E$ and sequenced BYMV isolates. Our results suggest that, in $P$. sativum, resistances mediated by genes $s b m l$ against PSbMV and $w l v$ against BYMV-W are caused by the same allele of $e I F 4 E$. Further, we have shown that a single amino acid change in VPg of BYMV-W is sufficient to overcome resistance mediated by $w l v$, and that this mutation does not compromise BYMV-W infection on $P$. sativum with the resistance gene mo.

\section{MATERIALS AND METHODS}

\section{$P$. sativum genotypes and BYMV isolates.}

The following $P$. sativum genotypes were used in this study: cvs. Bonneville (by courtesy of K. E. Keller, United States Department of Agriculture-Agricultural Research Service (USDAARS), Corvallis, OR, U.S.A.), JI1405 and JI2009 (by courtesy of A. J. Maule, John Innes Centre, England), DSP (Dæhnfeldt, Odense, Denmark), and Brutus (DLF-Trifolium, St. Heddinge, Denmark); and PI lines PI193586, PI193835, PI193836, PI269774, PI269818, PI347492, PI347329, PI347422, PI347464, and PI347484 (Western Regional Plant Introduction Station, Pullman, WA, U.S.A.).

The BYMV-S isolate (Guyatt et al. 1996) was provided by A. Davidson, Monash University, Australia. BYMV-W (Hampton et al. 1992) was provided by K. E. Keller, USDA-ARS. BYMV-S and BYMV-W particles were purified as described by (Olsen and Johansen 2001) from P. sativum Brutus and DSP, respectively, and used to raise polyclonal antibodies in rabbits. Antibodies were used in DAS-ELISA to assay plant tissue samples of $0.25 \mathrm{~g}$ for the presence of virus coat protein (Kohnen et al. 1995). In all experiments, at least four uninoculated $P$. sativum plants were included to determine the mean $\mathrm{A}_{405}$ reading of uninfected plant tissue.

\section{Cloning and sequencing of BYMV-W.}

Standard cloning procedures were employed (Sambrook et al. 1989). Restriction and modifying enzymes (Roche Diagnostics, Mannheim, Germany and New England Biolabs, Ipswich, MA, U.S.A), Taq DNA polymerase (GibcoBRL Roskilde, Denmark), Pfu DNA polymerase (Stratagene, La 
Jolla, CA, U.S.A.), and M-MLV reverse transcriptase (Invitrogen, Taastrup, Denmark) were used according to the manufacturer's instructions. PCR products were cloned using TOPOtechnology (Invitrogen). Oligonucleotide primers and sequencing analyses were delivered by MWG (Ebersberg, Germany).

RNA was extracted from virus particles and used as template for oligo(dT) primed cDNA synthesis by M-MLV reverse transcriptase. The cDNA was amplified by PCR using Taq DNA polymerase using a series of degenerate primers, which were designed on the basis of the BYMV-S sequence (U47033). PCR products were cloned, and sequences obtained from these clones covered nucleotides 26 to 2,400, 4,250 to 5,750 , and 6,100 to 8,200 . Based on these three sequences, primers were designed to obtain sequence of the $5^{\prime}$ terminus by $5^{\prime}$ rapid amplification of cDNA ends (RACE) (Clontech, Mountain View, CA, U.S.A.) and amplify cDNA to obtain a complete sequence. The 5' RACE gave sequences up to nucleotide 21 as predicted by comparison with BYMV-S (U47033) and BYMV-MB4 (NC 003492).

For assembly of a full-length clone of BYMV-W, the complete genome was amplified by PCR using $P f u$ DNA polymerase generating overlapping cDNA fragments using (forward/ reverse) primer pairs 5' GTCTAGAAAAAATATAAAAACCA ATCAAGAC/5' TGGTGTCTATTCTGATTCGGCTAC for nucleotides 1 to $2,453,5^{\prime}$ CCATCAGCGCAGGGTACCATG/5' GTCTGCAGCATCTCTGAACCTC for nucleotides 2,310 to 5,731, 5' CCTGAGTGTGTTGCACAGTGCAC/5' ATTTGGC GGTATGGAGGTAAACAC for nucleotides 5,404 to 6,808 , and 5' CTTTGTGAAGGGTGCAGGGC/5' GCGTCTAGATTTTT TTTTTTTTTTTTTTT for nucleotides 6,058 to poly(A). Sequences were obtained from at least two independent clones of these fragments and the BYMV-W sequence was submitted to the National Center for Biotechnology Information (NCBI) Nucleotide Sequence database, accession number DQ641248. Putative coding regions were predicted by comparison with BYMV-S and BYMV-MB4 (Guyatt et al. 1996; Nakamura et al. 1996) and the VPg coding region assigned to nucleotides 5,661 to 6,233 .

\section{Construction \\ of infectious clones pBYMV-W ${ }^{\mathrm{H} 116}$ and pCABYMV-W.}

The complete cDNA sequence of BYMV-W was assembled between the $35 \mathrm{~S}$ promoter and nopaline synthase (NOS) terminator in pAGUS1 in a series of standard cloning steps essentially as described by Johansen (1996). Nonviral nucleotides after the transcription start site were eliminated by mung bean nuclease (NEB, Ipswich, MA, U.S.A.) treatment. To stabilize the plasmid in Escherichia coli, intron IV from Solanum tuberosum gene ST-LS1 was inserted after nucleotide 5,729 to generate $\mathrm{pBYMV}-\mathrm{W}$. Virus derived from $\mathrm{pBYMV}-\mathrm{W}$ has the same infection profile on $P$. sativum genotypes as native BYMV-W (data not shown).

pBYMV-W $\mathrm{W}^{\mathrm{H} 116}$ was constructed by mutating $\mathrm{GC}$ at nucleotide 6,027 to 6,028 to AT, thus changing the predicted $\mathrm{VPg}$ codon 116 CGC (Arg) to CAT (His). Plasmids pBYMV and pBYMV-W ${ }^{\mathrm{H} 116}\left(0.5 \mu \mathrm{g} / \mu \mathrm{l}\right.$ in $\left.\mathrm{H}_{2} \mathrm{O}\right)$ were inoculated by mechanical inoculation by hand to P. sativum Brutus, and sap (leaf tissue homogenized in $0.03 \mathrm{M}$ phosphate buffer, $\mathrm{pH} 7.6$, with $4 \%$ polyethylenglycol) from these plants was used to inoculate test plants.

To allow agroinoculation of BYMV-W cDNA, the plasmid pCABYMV-W was generated by inserting the expression cassette from $\mathrm{pBYMV}-\mathrm{W}$, including $35 \mathrm{~S}$ promoter, BYMV-W cDNA, and NOS terminator, into the binary vector pCAMBIA1300 (Cambia, Canberra, Australia), which was modified by deletion of the hygromycin resistance gene (Constantin et al. 2004).
Sequencing of $e I F 4 E$ cDNA from $P$. sativum genotypes.

To obtain sequence information on $e I F 4 E$ from $P$. sativum, primers AD-FW: 5' CCCAAAAAAAGAGATCGAATTAGGA and AD-RV: 5' CTCTGCAGTAATACGACTCACTAT (flanking the inserts of $\lambda$-phage library vector pAD-Gal4-2-1 inserts) (Stratagene) and eIF-FW: 5' GAGTTTTGGAGCATTTACAA TAAC and eIF-RV: 5' CACAACAGCTCC(AG)CATATTTCAT (designed on the basis on predicted IF $4 E$ EST sequences from legumes) were used to amplify DNA fragments from a cDNA library of cv. Scout provided by A. J. Maule John Innes Centre, U.K. The two fragments amplified with primer pairs AD-FW: eIF-RV and eIF-FW:AD:RV were cloned using TOPO TA cloning kit (Invitrogen) and inserts were sequenced. The sequence differed at a single nucleotide at the fifth codon, which was GAC (Asp) in contrast to GAA (Glu) from the published sequence (ARR04332). Oligonucleotide 5' CAGTCAAAAGA TTATCCGCACACA annealing at the $3^{\prime}$ untranslated region (UTR) of $e I F 4 E$ was used to prime cDNA synthesis on RNA extracted from Brutus, Bonneville, DSP, PI269818, PI193835, and PI347464 using RNAeasy kit (Qiagen, Hilden, Germany). Two primers that annealed to the $5^{\prime}$ (5' GGCACGAGGAGAG AGCAAAAATG) and 3' (5' CGGAGAGGCATCTCATGCAA) UTR were used to PCR amplify the region coding eIF4E by Taq DNA polymerase. The PCR fragments were TOPO cloned and sequenced. The sequence of eIF4E from PI269818 (AY611425) and PI193835 (AY611423) were published by Gao and associates (2004b); however, we consistently found the fifth codon of the open reading frame to be GAC. The sequences of eIF4E from Brutus, Bonneville, DSP, PI347464, and PI193835 were submitted to the NCBI Nucleotide Sequence database and assigned accession numbers DQ641470, DQ641471, DQ641472, DQ641473, and DQ641474.

\section{Construction of pCAPE2-R-eIF4E and pCAPE2-S-eIF4E.}

The PEBV vector is composed of two binary plasmids, pCAPE1 and pCAPE2, carrying cDNA of PEBV RNA1 and a modified RNA2 for insertion of heterologous fragments (Constantin et al. 2004). The 684-nucleotide coding region of eIF4E from BYMV-W-susceptible (JI2009) and -resistant (PI193835) $P$. sativum genotypes were amplified by PCR using $P f u$ DNA polymerase with primers 5' GCCCATGGTTGTAGA AGACACCCCCAAATCC and 5' GTTACACAACATATTTGT TTTTAGCATTTC). The fragments were TOPO cloned and sequenced. Binary vectors pCAPE2-R-eIF4E and pCAPE2-SeIF4E were constructed by replacing the GFP coding region of pCAPE2-GFP (Constantin et al. 2004) with the coding region of $e I F 4 E$ from PI193835 and JI2009, respectively.

\section{Functional complementation assay by PEBV-vectored eIF4E.}

Agrobacterium sp. strain GV3101 was transformed with pCABYMV-W, pCAPE2-R-eIF4E, pCAPE2-S-eIF4E, and cultures for agroinoculation were grown as described in Constantin and associates (2004). Optical density at $550 \mathrm{~nm}$ $\left(\mathrm{OD}_{550}\right)$ was determined on overnight cultures, and bacteria were harvested by centrifugation and resuspended in $100 \mu \mathrm{l}$ of infiltration medium $\left(10 \mathrm{mM} \mathrm{NaCl}, 1.75 \mathrm{mM} \mathrm{CaCl}_{2}, 100 \mu \mathrm{M}\right.$ acetosyringone) per milliliter of culture per 1.0 unit at $\mathrm{OD}_{550}$. For inoculation, infiltration cultures of pCABYMV-W, pCAPE1, and pCAPE2-derivative (GFP or R-eIF4E or SeIF4E) were mixed $1: 1: 1$ and infiltrated to four leaves of BYMV-W-susceptible (Bonneville) and -resistant (PI193835) $P$. sativum plants. The experiment was repeated three times and, in each experiment, at least six plants were used per treatment. In the first experiment, inoculated leaves were assayed by DAS-ELISA for the presence of BYMV coat protein $5 \mathrm{dpi}$. In the second, third, and fourth experiments, inoculated leaves 
were assayed for the presence of virus coat protein at $8 \mathrm{dpi}$ and uninoculated leaves at 3 wpi.

\section{Sequence analysis of the VPg coding region.}

To determine the sequence of the VPg coding region of virus in infected plants, RNA was extracted from leaf tissue and the VPg coding region was amplified by RT-PCR and sequenced as described by Borgstrøm and Johansen (2001) using primers Fw-VPg (5' GCGTACGAAACAGAAACTGAGGTTCAGAG) and Rv-VPg (5' TCTCGAGTAAGCAAACATTTGAAGC). For analysis of the VPg coding region in leaves agroinoculated with pCABYMV, RNA was treated with RQ1 RNAse-free DNAse (Promega Corps., Madison, WI, U.S.A.) to degrade DNA inoculum. RT-PCR products amplified by Taq DNA polymerase were analyzed by agarose gel electrophoresis either undigested or after digestion with restriction enzyme HaeII (Roche Diagnostics). As a control of RNA extractions, a fragment of $P$. sativum PDS was amplified by RT-PCR using primers 5' ATTGGGCGGTGAACTCCATCTTAATTC and 5' GAT CTGCAGAAATTTCATCAGGG.

\section{ACKNOWLEDGMENTS}

This work was supported by grants 9702802 and 53-00-0330 from the Danish Agricultural and Veterinary Research Council. We thank B. Olsen and M. Læssøe Steffensen for expert technical assistance with laboratory work and maintenance of plants in the greenhouse.

\section{LITERATURE CITED}

Albar, L., Bangrantz-Reyser, M., Hébrard, E., Ndjiondjop, M.-N., Jones, M., and Ghesquire, A. 2006. Mutations in the eIF(iso)4G translation initiation factor confer high resistance of rice to Rice yellow mottle virus. Plant J. 47:417-426.

Ayme, V., Souche, S., Caranta, C., Jacquemond, M., Chadæuf, J., Palloix, A., and Moury, B. 2006. Different mutations in the genome-linked protein VPg of Potato virus $Y$ confer virulence on the $p v r 2^{3}$ resistance in pepper Mol. Plant-Microbe Interact. 19:557-563.

Beauchemin, C., Boutet, N., and Laliberté, J.-F. 2007. Visualization of the interaction between the precursors of $\mathrm{VPg}$, the viral protein linked to the genome of Turnip mosaic virus, and the translation eukaryotic initiation factor iso 4E in planta. J. Virol. 81:775-782.

Borgstrøm, B., and Johansen, I. E. 2001. Mutations in pea seedborne mosaic virus genome-linked protein VPg after pathotype-specific virulence in Pisum sativum. Mol. Plant-Microbe Interact. 14:707-714.

Constantin, G. D., Krath, B. N., MacFarlane, S. A., Nicolaisen, M., Johansen, I. E., and Lund, O. S. 2004. Virus-induced gene silencing as a tool for functional genomics in a legume species. Plant J. 40:622-631.

Gao, Z., Eyers, S., Thomas, C., Ellis, N., and Maule, A. 2004a. Identification of markers tightly linked to $\mathrm{sbm}$ recessive genes for resistance to Pea seed-borne mosaic virus. Theor. Appl. Genet. 109:488-494.

Gao, Z., Johansen, E., Eyers, S., Thomas, C. L., Ellis, T. H. N., and Maule, A. J. 2004b. The Potyvirus recessive resistance gene, sbm1, identifies a novel role for translation initiation factor eIF4E in cell-to-cell trafficking. Plant J. 40:376-385.

Guyatt, K. J., Proll, D. F., Menssen, A., and Davidson, A. D. 1996. The complete nucleotide sequence of bean yellow mosaic potyvirus RNA. Arch. Virol. 141:1231-1246.

Hampton, R. O., Shukla, D. D., and Jordan, R. L. 1992. Comparative potyvirus host range, serology, and coat protein peptide profiles of white lupin mosaic virus. Phytopathology 82:566-571.

Hjulsager, C. K., Lund, O. S., and Johansen, I. E. 2002. A new pathotype of Pea seedborne mosaic virus explained by properties of the P3-6k1and viral genome-linked protein (VPg)-coding regions. Mol. PlantMicrobe Interact. 15:169-171.

Johansen, I. E. 1996. Intron insertion facilitates amplification of cloned virus cDNA in Escherichia coli while biological activity is reestablished after transcription in vivo. Proc. Natl. Acad. Sci. U.S.A. 93:12400-12405.

Johansen, I. E., Lund, O. S., Hjulsager, C. K., and Laursen, J. 2001. Recessive resistance in Pisum sativum and potyvirus pathotype resolved in a gene-for-cistron correspondence between host and virus. J. Virol. 75:6609-6614.

Kang, B.-C., Yeam, I., Frantz, J. D., Murphy, J. F., and Jahn, M. M. 2005. The pvrl locus in Capsicum encodes a translation initiation factor
eIF4E that interacts with Tobacco etch virus VPg. Plant J. 42:392-405.

Kohnen, P. D., Johansen, I. E., and R. O. Hampton. 1995. Characterization and molecular detection of the $\mathrm{P} 4$ pathotype of pea seedborne mosaic potyvirus. Phytopathology 85:789-793.

Kuhne, T., Shi, N., Proeseler, G., Adams, M. J., and Kanyuka, K. 2003. The ability of a bymovirus to overcome the rym4-mediated resistance in barley correlates with a codon change in the VPg coding region on RNA1. J. Gen. Virol. 84:2853-2859.

Marx, G. A., and Provvidenti, R. 1979. Linkage relations of mo. Pisum Newsl. 11:28-29.

Moury, B., Morel, C., Johansen, E., Guilbaud, L., Souche, S., Ayme, V., Caranta, C., Palloix, A., and Jacquemond, M. 2004. Mutations in Potato virus $Y$ genome-linked protein determine virulence towards recessive resistances in Capsicum annuum and Lycopersicon hirsutum. Mol. Plant-Microbe Interact. 17:322-329.

Nakamura, S., Honkura, R., Iwai, T., Ugaki, M., and Ohashi, Y. 1996. The complete nucleotide sequence of bean yellow mosaic virus genomic RNA. Ann. Phytopathol. Soc. Jpn. 62:472-477.

Nicaise, V., German-Retana, S., Sanjuán, R., Dubrana, M. P., Mazier, M., Maisonneuve, B., Candresse, T., Caranta, C., and LeGall, O. 2003. The eukaryotic translation initiation factor $4 \mathrm{E}$ controls lettuce susceptibility to the potyvirus Lettuce mosaic virus. Plant Physiol. 132:1272-1282.

Nicolas, O., Dunnington, S. W., Gotow, L. F., Pirone, T. P., and Hellmann, G. M. 1997. Variations in the VPg protein allow a potyvirus to overcome va gene resistance in tobacco. Virology 237:452-459.

Olsen, B. S., and Johansen, I. E. 2001. Nucleotide sequence and infectious cDNA clone of the L1 isolate of Pea seed-borne mosaic potyvirus. Arch. Virol. 146:15-25.

Provvidenti, R. 1987. Inheritance of resistance to clover yellow vein virus in Pisum sativum. J. Hered. 78:126-128.

Provvidenti, R., and Alconero, R. 1988. Inheritance of resistance to a lentil strain of pea seed-borne mosaic virus in Pisum sativum. J. Hered. 79:45-47

Provvidenti, R., and Hampton, R. O. 1991. Chromosomal distribution of genes for resistance to seven potyviruses in Pisum sativum. Pisum Genet. 23:26-28.

Provvidenti, R., and Hampton, R. O. 1993. Inheritance of resistance to White lupin mosaic virus in common pea. HortScience 28:836-837.

Rajamäki, M.-L., and Valkonen, J. P. T. 1999. The 6 K 2 protein and the VPg of Potato Virus A are determinants of systemic infection in Nicandra physaloides. Mol. Plant-Microbe Interact. 12:1074-1081.

Randles, J. W., Davies, C., Gibbs, A. J., and Hatta, T. 1980. Amino acid composition of capsid protein as a taxonomic criterion for classifying the atypical S strain of bean yellow mosaic virus. Aust. J. Biol. Sci. 33:245-54.

Revers, F., Le Gall, O., Candresse, T., and Maule, A. J. 1999. New advances in understanding the molecular biology of plant/potyvirus interactions. Mol. Plant-Microbe Interact. 12:367-376.

Robaglia, C., and Caranta, C. 2006. Translation initiation factors: A weak link in plant virus infection. Trends Plant Sci. 11:40-45.

Ruffel, S., Dussault, M.-H., Palloix, A., Moury, B., Bendahmane, A., Robaglia, C., and Caranta, C. 2002. A natural recessive resistance gene against Potato virus $Y$ in pepper corresponds to the eukaryotic initiation factor 4E (eIF4E). Plant J. 32:1067-1075.

Ruffel, S., Gallois, J.-L., Moury, B., Robaglia, C., Palloix, A., and Caranta, C. 2006. Simultaneous mutations in translation initiation factors eIF4E and $\mathrm{eIF}$ (iso) $4 \mathrm{E}$ are required to prevent pepper veinal mottle virus infection of pepper. J. Gen. Virol. 87:2089-2098.

Sambrook, J., Fritsch, E. F., and Maniatis, T. A. 1989. Molecular Cloning: A Laboratory Manual, 2nd ed. Cold Spring Harbor Laboratory Press, Cold Spring Harbor, NY, U.S.A.

Schaad, M. C., Lellis, A. D., and Carrington, J. C. 1997. VPg of tobacco etch potyvirus is a host genotype-specific determinant for long distance movement. J. Virol. 71:8624-8631.

Schroeder, W. T., and Provvidenti, R. 1971. A common gene for resistance to bean yellow mosaic virus and watermelon mosaic virus 2 in Pisum sativum. Phytopathology 61:846-848.

Shukla, D. D., Ward, C. W., and Brunt, A. A. 1994. The Potyviridae. CAB International, Wallingford, U.K.

Stein, N., Perovic, D., Kumlehn, J., Pellio, B., Stracke, S., Streng, S., Ordon, F., and Graner, A. 2005. The eukaryotic translation initiation factor 4E confers multiallelic recessive Bymovirus resistance in Hordeum vulgare (L.). Plant J. 42:912-922.

Yen, D. E., and Fry, P. R. 1956. The inheritance of immunity to pea mosaic virus. Aust. J. Agric. Res. 7:272-281.

Yoshii, M., Nishikiori, M., Tomita, K., Yoshioka, N., Kozuka, R., Naito, S., and Ishikawa, M. 2004 The Arabidopsis Cucumovirus Multiplication 1 and 2 loci encode translation initiation factors $4 \mathrm{E}$ and $4 \mathrm{G}$. J. Virol. 78:6102-6111. 